\title{
Outage performance of underlay cognitive radio networks over mix fading environment
}

\author{
Nguyen Hong Nhu', Cuu Ho Van², Van-Duc Phan ${ }^{3}$, Tan N. Nguyen ${ }^{4}$, Miroslav Voznak, \\ Jaroslav Zdralek ${ }^{6}$ \\ ${ }^{1,2}$ Faculty Electronics and Telecommunications, Saigon University, Ho Chi Minh City, Vietnam \\ ${ }^{3}$ Faculty of Automobile Technology, Van Lang University, Ho Chi Minh City, Vietnam \\ ${ }^{4}$ Wireless Communications Research Group, Faculty of Electrical and Electronics Engineering, \\ Ton Duc Thang University, Ho Chi Minh City, Vietnam \\ 1,4,5,6 VSB-Technical University of Ostrava, Ostrava-Poruba, Czech Republic
}

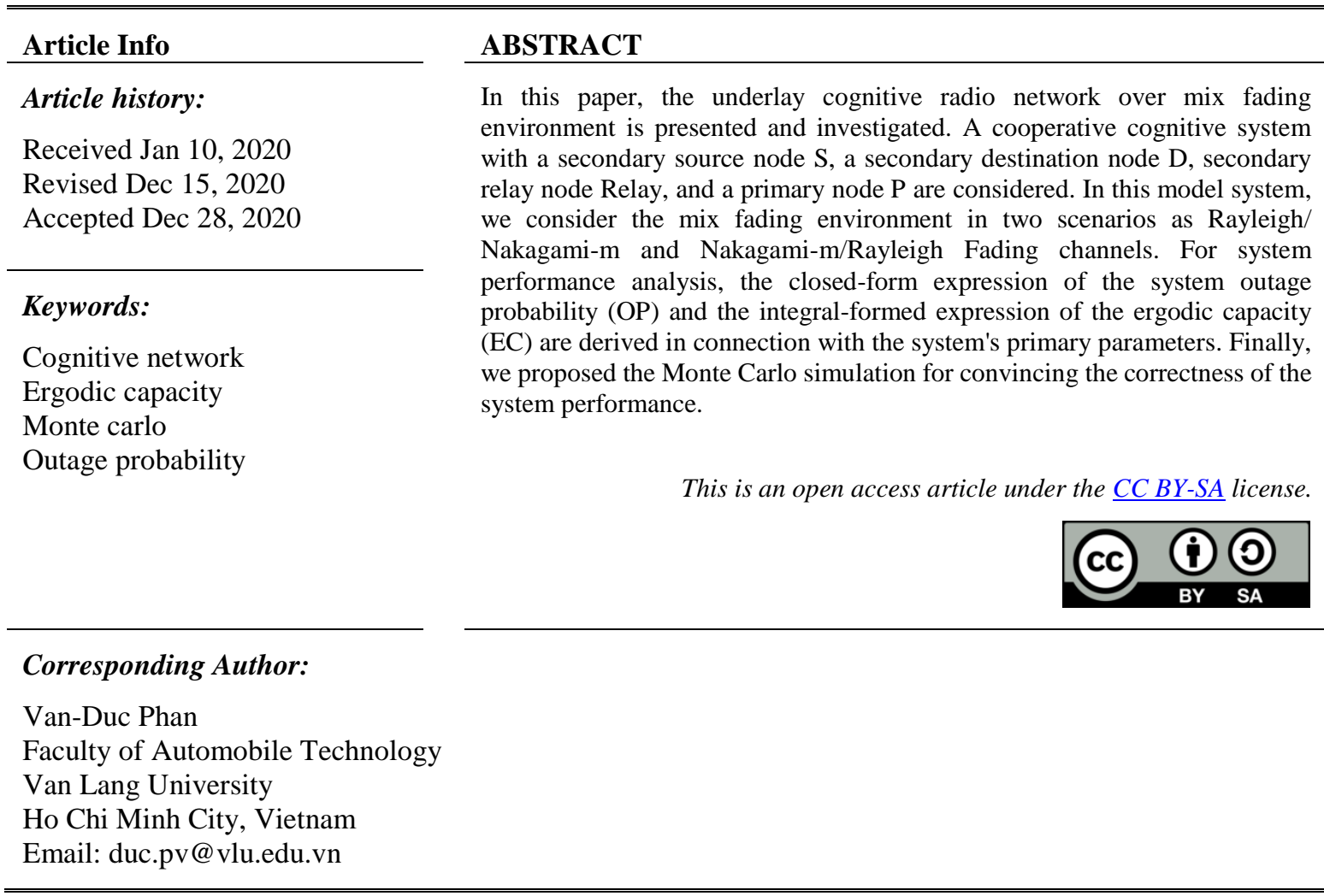

\section{INTRODUCTION}

In underlay CR (also called as spectrum sharing), a secondary user is allowed to access spectrum at any time as long as the received interference at a primary user is regulated be-low a predetermined level, i.e., interference temperature [1-12]. Due to long-distance and deep fading, a signal received at a destination may not be decoded correctly. To overcome this problem, the cooperative relay has been incorporated to transfer signals from source to destination successfully via intermediate relays. In [13], the exact closed-form expression for the outage probability of cognitive radio dual-hop amplify-and-forward relay networks is studied. The authors in [14] considered the outage performance of decode-and-forward relaying in cognitive radio networks over Rayleigh fading channels, subject to the relay location for a secondary user and the spectrum sharing of the secondary system with multiple primary transceivers, where the secondary users communicate via an energy harvesting decode-and-forward relay under the primary outage constraint is proposed in [15]. Furthermore, the performance of a multi-hop cognitive relay network, which harvests energy from a PB using a TSR protocol is investigated in [16] and authors in [17] investigated a hybrid CR system that probabilistically switches the spectrum access modes between the overlay and underlay CR modes for an increase of secondary user's throughput. 
In this paper, the underlay cognitive radio network over mix fading environment is presented and investigated. A cooperative cognitive system with a secondary source node $\mathrm{S}$, a secondary destination node $\mathrm{D}$, secondary relay node Relay, and a primary node $\mathrm{P}$ are considered. In this model system, we consider the mix fading environment in two scenarios as Rayleigh/Nakagami-m and Nakagami-m/Rayleigh Fading channels. For system performance analysis, the closed-form expression of the system outage probability (OP) and the integral-formed expression of the ergodic capacity (EC) are derived in connection with the system's primary parameters. Finally, we proposed the Monte Carlo simulation for convincing the correctness of the system performance.

The rest of this manuscript can be formulated as the following. The system model of the underlay cognitive radio network over mix fading environment is drawn in the second section. The system performance in term of the system OP and EC is derived in the thirst section. Then, some numerical results and discussions is given in the fourth section. Finally, the last section concluded the manuscript.

\section{SYSTEM MODEL}

A cooperative cognitive system, as shown in Figure 1, comprising of a secondary source node S, a secondary destination node $\mathrm{D}$, secondary relay node Relay, and a primary node $\mathrm{P}$ are considered. Assumed that all nodes are equipped single antenna, operated in half-duplex mode, and are the mobile nodes. Secondary nodes $\mathrm{S}$ and $\mathrm{D}$ are assumed to lack a direct link and relay is a bridge where connect the communication for $\mathrm{S}$ and D. In our model, the decode and forward (DF) technique is employed. Hence, during the first time slot, $S$ will broadcast its signal to relay [18-21].

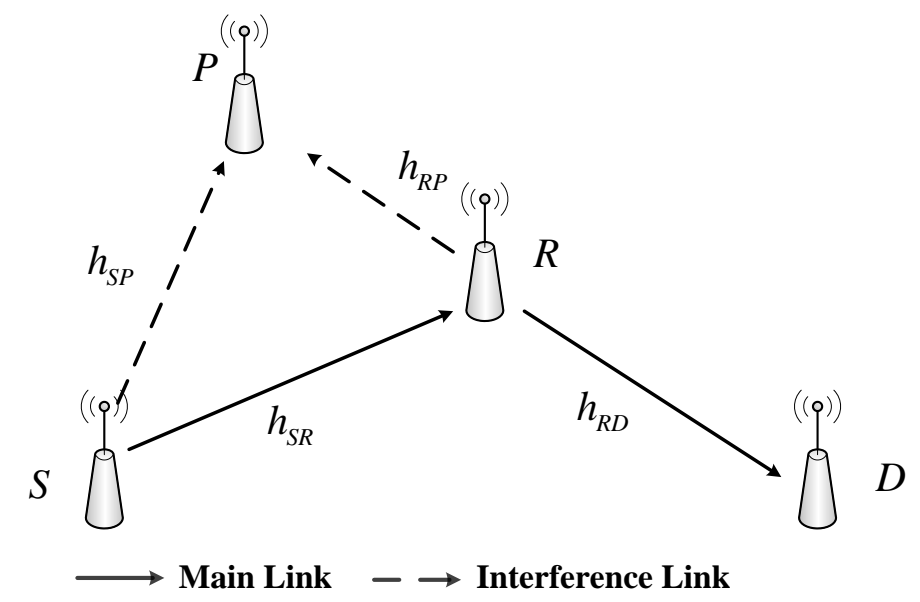

Figure 1. $\mathrm{P}_{\text {out }}$ versus the transmit power $\mathrm{P} 3$ with different $\mathrm{P}_{1}$ and $\mathrm{P}_{2}\left(\mathrm{P}_{1}, \mathrm{P}_{2}\right)$

So, the received signal at the relay can be expressed by

$$
y_{R}=h_{S R} x_{s}+n_{R}
$$

In the second time slot, the relay $\mathrm{R}$ will decode successfully $x_{s}$ and then forward to $\mathrm{D}$. Therefore, the received signal at the destination can be given as

$$
\begin{aligned}
& y_{D}=h_{R D} x_{R}+n_{D} \\
& \mathrm{E}\left\{\left|x_{R}\right|^{2}\right\}=P_{R}
\end{aligned}
$$

As [22], the transmit power at the $\mathrm{S}$ and $\mathrm{R}$ can be obtained as, respectively:

$$
P_{s}=\frac{I_{P}}{\left|h_{S P}\right|^{2}}
$$


where $h_{S P}$ is the channel fading coefficient of S-P link.

$$
P_{R}=\frac{I_{P}}{\left|h_{R P}\right|^{2}}
$$

Where $h_{R P}$ is the channel fading coefficient of R-P link.

The signal to noise ratio (SNR) at the R and D can be computed from (1) and (2), then substituting (3) and (4), respectively:

$$
\gamma_{S R}=\frac{P_{s}\left|h_{S R}\right|^{2}}{N_{0}}=\frac{\Psi\left|h_{S R}\right|^{2}}{\left|h_{S P}\right|^{2}}=\frac{\Psi X}{Y}
$$

where $\Psi=\frac{I_{P}}{N_{0}}, X=\left|h_{S R}\right|^{2}$ and $Y=\left|h_{S P}\right|^{2}$

$$
\gamma_{R D}=\frac{P_{R}\left|h_{R D}\right|^{2}}{N_{0}}=\frac{\Psi\left|h_{R D}\right|^{2}}{\left|h_{R P}\right|^{2}}=\frac{\Psi Z}{T}
$$

where $Z=\left|h_{R D}\right|^{2}$ and $T=\left|h_{R P}\right|^{2}$. From (5) and (6), the end to end SNR of the secondary DF system can be expressed as

$$
\gamma_{D F}=\min \left(\gamma_{S R}, \gamma_{R D}\right)
$$

\section{OUTAGE PROBABILITY (OP) ANALYSIS}

3.1. Outage probability $(\mathrm{OP})$ analysis

The OP can be defined by

$$
O P=\operatorname{Pr}\left(\gamma_{D F}<\gamma_{t h}\right)
$$

where $\gamma_{t h}$ is a predetermined SNR threshold. Substituting (5) and (6) into (8), the OP can be obtained as

$$
\begin{aligned}
O P & =\operatorname{Pr}\left\{\min \left(\gamma_{S R}, \gamma_{R D}\right)<\gamma_{t h}\right\}=\operatorname{Pr}\left\{\min \left(\frac{\Psi X}{Y}, \frac{\Psi Z}{T}\right)<\gamma_{t h}\right\} \\
& =1-\operatorname{Pr}\left(\frac{\Psi X}{Y} \geq \gamma_{t h}\right) \operatorname{Pr}\left(\frac{\Psi Z}{T} \geq \gamma_{t h}\right)
\end{aligned}
$$

a. Scenario 1: $h_{S R}, h_{R D}$ are Rayleigh fading channel and $h_{S P}, h_{R P}$ are Nakagami-m fading channel.

As [23], the probability density function (PDF) and the cumulative distribution function (CDF) of X, $\mathrm{Z}$ and $\mathrm{Y}, \mathrm{T}$ can be given by, respectively:

$$
\begin{aligned}
& f_{i}(x)=\frac{1}{\lambda_{j}} e^{-\frac{x}{\lambda_{j}}} \\
& F_{i}(x)=1-e^{-\frac{x}{\lambda_{j}}}
\end{aligned}
$$

where $i \in(X, Z), j \in(S R, R D)$ and $\lambda_{j}$ is the mean of random variables (RVs) $\mathrm{X}, \mathrm{Z}$.

Moreover, we also have:

$$
f_{a}(x)=\frac{x^{m-1}}{(m-1) !\left(\Omega_{a}\right)^{m}} \exp \left(-\frac{x}{\Omega_{a}}\right)
$$




$$
F_{a}(x)=1-\exp \left(-\frac{x}{\Omega_{a}}\right) \sum_{t=0}^{m-1} \frac{x^{t}}{t !\left(\Omega_{a}\right)^{t}}
$$

where $a \in(Y, T), \Omega_{a}=\frac{\lambda_{b}}{m}$ in which $b \in(S P, R P)$ and $m$ is the Nakagami-m parameter and $\lambda_{b}$ is the mean of RVs Y, T

From (9), we have:

$$
\begin{aligned}
\operatorname{Pr}\left(\frac{\Psi X}{Y} \geq \gamma_{t h}\right) & =1-\operatorname{Pr}\left(\frac{\Psi X}{Y}<\gamma_{t h}\right)=1-\operatorname{Pr}\left(X<\frac{\gamma_{t h} Y}{\Psi}\right) \\
& =1-\int_{0}^{\infty} F_{X}\left(\frac{\gamma_{t h} y}{\Psi} \mid Y=y\right) \times f_{Y}(y) d y
\end{aligned}
$$

Substituting (11) and (12) into (14), we can obtain:

$$
\begin{aligned}
& \operatorname{Pr}\left(\frac{\Psi X}{Y} \geq \gamma_{t h}\right)=\int_{0}^{\infty} \frac{y^{m-1}}{(m-1) !\left(\Omega_{Y}\right)^{m}} \exp \left(-\frac{y}{\Omega_{Y}}-\frac{\gamma_{t h} y}{\Psi \lambda_{S R}}\right) d x \\
& =\frac{1}{(m-1) !\left(\Omega_{Y}\right)^{m}} \int_{0}^{\infty} y^{m-1} \exp \left(-y\left[\frac{1}{\Omega_{Y}}+\frac{\gamma_{t h}}{\Psi \lambda_{S R}}\right]\right) d y
\end{aligned}
$$

where $\Omega_{Y}=\frac{\lambda_{S P}}{m}$

Applying $(3.381,4)$ of [24], (15) can be claimed by (16):

$$
\operatorname{Pr}\left(\frac{\Psi X}{Y} \geq \gamma_{t h}\right)=\frac{\Gamma(m)\left(\frac{1}{\Omega_{Y}}+\frac{\gamma_{t h}}{\Psi \lambda_{S R}}\right)^{-m}}{(m-1) !\left(\Omega_{Y}\right)^{m}}=\left(1+\frac{\gamma_{t h} \Omega_{Y}}{\Psi \lambda_{S R}}\right)^{-m}
$$

Similar, we have:

$$
\operatorname{Pr}\left(\frac{\Psi Z}{T} \geq \gamma_{t h}\right)=\left(1+\frac{\gamma_{t h} \Omega_{T}}{\Psi \lambda_{S R}}\right)^{-m}
$$

Substituting (16) and (17) into (9), the OP can be obtained as

$$
O P=1-\left\{\left(1+\frac{\gamma_{t h} \Omega_{Y}}{\Psi \lambda_{S R}}\right) \times\left(1+\frac{\gamma_{t h} \Omega_{T}}{\Psi \lambda_{S R}}\right)\right\}^{-m}
$$

b. Scenario 2: $h_{S R}, h_{R D}$ are Nakagami-m fading channel and $h_{S P}, h_{R P}$ are Rayleigh fading channel

Similar proof as above, so, substituting (10) and (13) into (14), and then applying (3.381,4) of [24], we can obtain:

$$
\begin{aligned}
\operatorname{Pr}\left(\frac{\Psi X}{Y} \geq \gamma_{t h}\right) & =\sum_{t=0}^{m-1} \frac{\left(\gamma_{t h}\right)^{t}}{t !\left(\Omega_{X} \Psi\right)^{t} \lambda_{S P}} \int_{0}^{\infty} y^{t} \exp \left(-y\left[\frac{\gamma_{t h}}{\Psi}+\frac{1}{\lambda_{S P}}\right]\right) d y \\
& =\sum_{t=0}^{m-1} \frac{\left(\gamma_{t h}\right)^{t}}{\left(\Omega_{X} \Psi\right)^{t} \lambda_{S P}} \times\left[\frac{\gamma_{t h}}{\Psi}+\frac{1}{\lambda_{S P}}\right]^{-t-1}
\end{aligned}
$$

where $\Omega_{X}=\frac{\lambda_{S R}}{m}$

Next, 


$$
\operatorname{Pr}\left(\frac{\Psi Z}{T} \geq \gamma_{t h}\right)=\sum_{t=0}^{m-1} \frac{\left(\gamma_{t h}\right)^{t}}{\left(\Omega_{Z} \Psi\right)^{t} \lambda_{R P}} \times\left[\frac{\gamma_{t h}}{\Psi}+\frac{1}{\lambda_{R P}}\right]^{-t-1}
$$

where $\Omega_{Z}=\frac{\lambda_{R D}}{m}$

Finally, substituting (19) and (20) into (9), the OP in this case can be claimed by

$$
\begin{aligned}
& O P=1-\sum_{t=0}^{m-1} \frac{\left(\gamma_{t h}\right)^{t}}{\left(\Omega_{X} \Psi\right)^{t} \lambda_{S P}} \times\left[\frac{\gamma_{t h}}{\Psi}+\frac{1}{\lambda_{S P}}\right]^{-t-1} \\
& \times \sum_{t=0}^{m-1} \frac{\left(\gamma_{t h}\right)^{t}}{\left(\Omega_{Z} \Psi\right)^{t} \lambda_{R P}} \times\left[\frac{\gamma_{t h}}{\Psi}+\frac{1}{\lambda_{R P}}\right]^{-t-1} \\
& =1-\sum_{t=0}^{m-1} \sum_{n=0}^{m-1} \frac{\left(\gamma_{t h}\right)^{t+n}}{\left(\Omega_{X}\right)^{t}\left(\Omega_{Z}\right)^{n}(\Psi)^{t+n} \lambda_{S P} \lambda_{R P}} \\
& {\left[\frac{\gamma_{t h}}{\Psi}+\frac{1}{\lambda_{S P}}\right]^{-t-1}\left[\frac{\gamma_{t h}}{\Psi}+\frac{1}{\lambda_{R P}}\right]^{-n-1}}
\end{aligned}
$$

\subsection{Ergodic capacity (EC) analysis}

The EC of the system can be defined as [25]

$$
C_{D F}=\frac{1}{\ln 2} \int_{0}^{\infty} \frac{1-F_{\gamma_{D F}}(x)}{1+x} d x
$$

a. Scenario 1:

Using result from (18) and replacing $\gamma_{t h}=x$, we have:

$$
F_{\gamma_{D F}}(x)=1-\left\{\left(1+\frac{x \Omega_{Y}}{\Psi \lambda_{S R}}\right) \times\left(1+\frac{x \Omega_{T}}{\Psi \lambda_{S R}}\right)\right\}^{-m}
$$

Substituting (23) into (22), we obtain:

$$
C_{D F}=\frac{1}{\ln 2} \int_{0}^{\infty} \frac{\left\{\left(1+\frac{x \Omega_{Y}}{\Psi \lambda_{S R}}\right) \times\left(1+\frac{x \Omega_{T}}{\Psi \lambda_{S R}}\right)\right\}^{-m}}{1+x} d x
$$

b. Scenario 2:

Similar to above, by using the (21) and then combining with (22), we claim:

$$
\begin{aligned}
& C_{D F}=\frac{1}{\ln 2} \times \sum_{t=0}^{m-1} \sum_{n=0}^{m-1} \frac{1}{\left(\Omega_{X}\right)^{t}\left(\Omega_{Z}\right)^{n}(\Psi)^{t+n} \lambda_{S P} \lambda_{R P}} \times \\
& \int_{0}^{\infty} \frac{x^{t+n} \times\left[\frac{x}{\Psi}+\frac{1}{\lambda_{S P}}\right]^{-t-1} \times\left[\frac{x}{\Psi}+\frac{1}{\lambda_{R P}}\right]^{-n-1}}{1+x} d x
\end{aligned}
$$

\section{NUMERICAL RESULTS AND DISCUSSION}

In this section, the Monte Carlo Simulation is conducted to convince the mathematical, analytical expressions in the above section [26-30]. The system OP versus $\psi$ is illustrated in Figure 2 with the main system parameters as $\mathrm{m}=3, \gamma_{\mathrm{th}}=3$ for both scenarios 1 and 2. As shown in Figure 2, the system OP falls intensively with rising of $\psi$ from $-5 \mathrm{~dB}$ to $20 \mathrm{~dB}$, and the system OP in the first scenario is better than in the second scenario. Moreover, the system EC versus $\psi$ is presented in Figure 3 with $m=3$ for both scenarios. In contrast with the above case, the system EC rises significantly, with a rising of $\psi$ from $-5 \mathrm{~dB}$ to $20 \mathrm{~dB}$, and the 
system EC in the second scenario is better than the first one. In Figures 2 and 3, the simulation and analytical curves agree well with each other for convincing the correctness of the above analytical section.

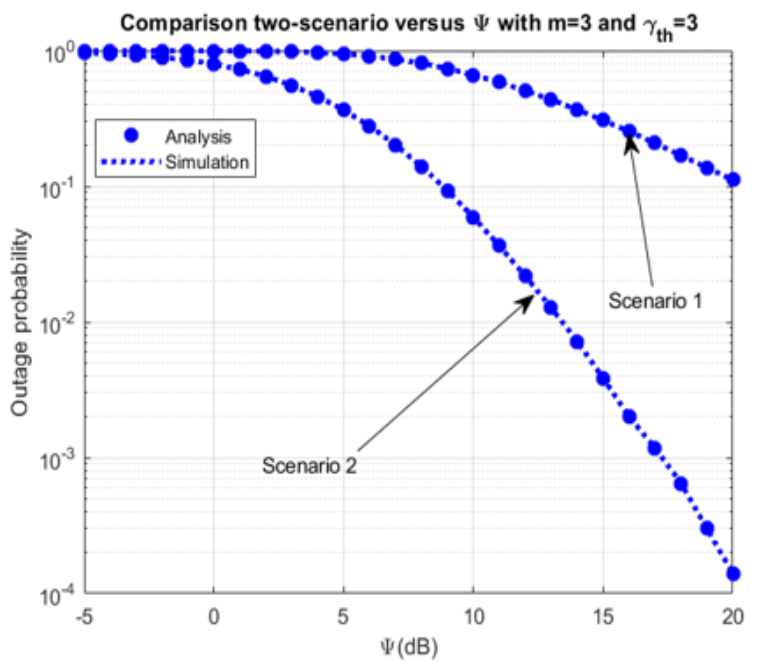

Figure 2. OP versus $\psi$

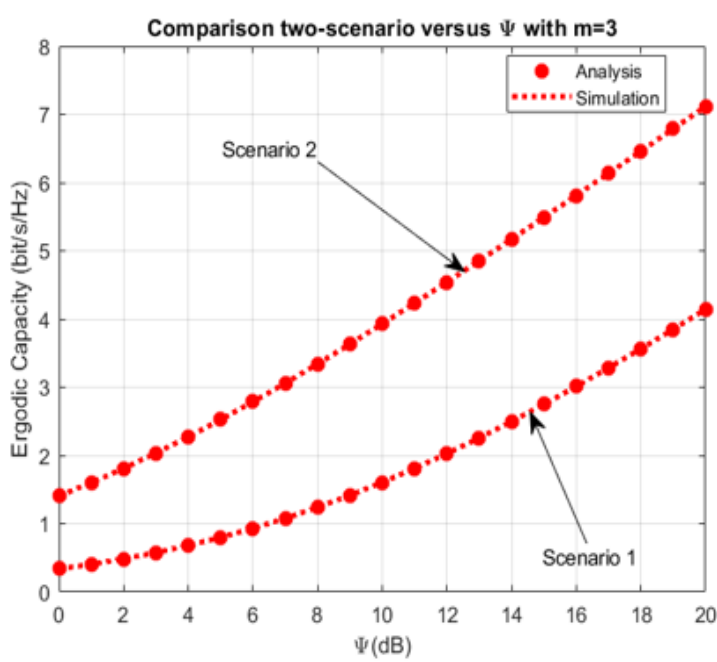

Figure 3. EC versus $\psi$

The influence of the Nakagami-m parameter on the system OP and EC are drawn in Figures 4 and 5, respectively. Here we set $\psi=10 \mathrm{~dB}$ and $\gamma_{\mathrm{th}}=1.5$ for both scenarios, as shown in Figures 4 and 5 . From Figure 4, we can see that the system OP in the first scenario has a slight increase, but the system OP in the second scenario falls intensively with the rising of Nakagami-m parameter from 1 to 10. From that result, we can conclude that the system performance in the second scenario is better than the first one. In the same way, the system EC in the second scenario is better than the first one, while the Nakagami-m parameter varies from 1 to 10, as shown in Figure 5. In Figures 4 and 5, the analytical curves overlap the simulation curves.

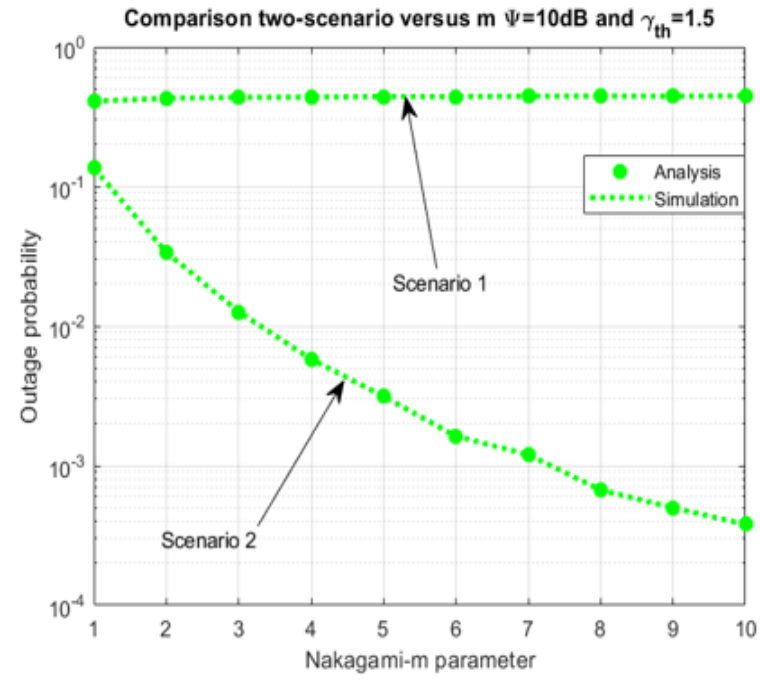

Figure 4. OP versus Nakagami-m parameter

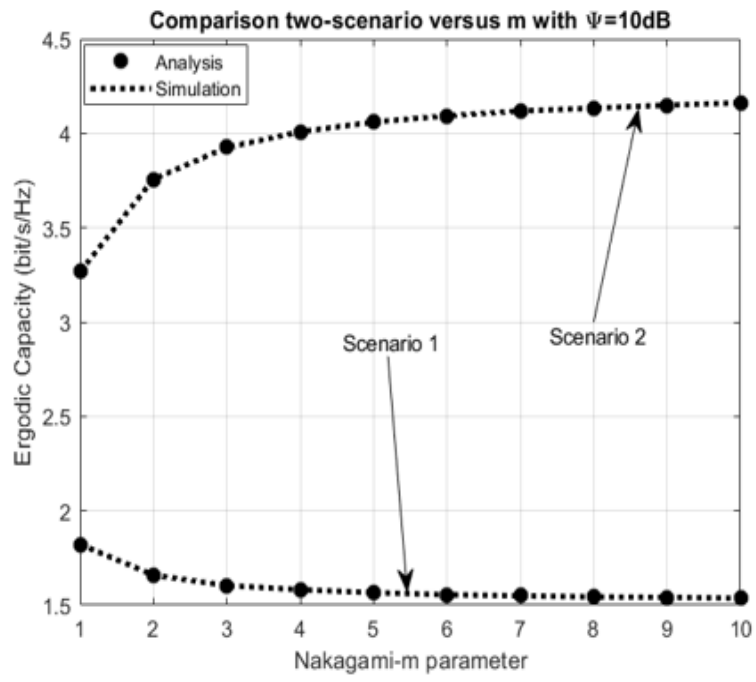

Figure 5. EC versus Nakagami-m parameter

Finally, the system OP versus $\gamma_{\text {th }}$ is illustrated in Figure 6 with $\mathrm{m}=3$, and $\psi=5 \mathrm{~dB}$. From Figure 6 , the results show that the system OP increases with the rising of the $\gamma_{\text {th }}$ from 0 to 6 . And once again, the system OP in the second Scenarios is better than the first one, and the analytical and simulation results are the same with each other to convince the correctness of the above analytical section. 


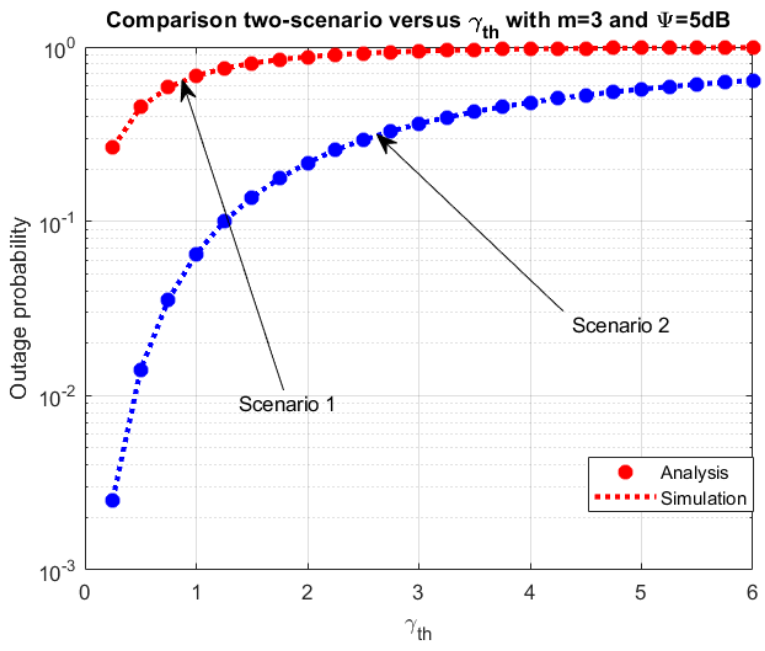

Figure 6. OP versus $\gamma_{\text {th }}$

\section{CONCLUSION}

In this paper, the underlay cognitive radio network over mix fading environment is presented and investigated. For system performance analysis, the closed-form expression of the system outage probability (OP) and the integral-formed expression of the ergodic capacity (EC) are derived in connection with the system's primary parameters. Finally, we proposed the Monte Carlo simulation for convincing the performance correctness. From the results, we can state that the analytical and simulation results overlap for both scenarios, and the second scenario is better in the system performance than the first scenario.

\section{REFERENCES}

[1] S. Haykin, "Cognitive radio: brain-empowered wireless communications," IEEE Journal on Selected Areas in Communications, vol. 23, no. 2, pp. 201-220, 2005, doi: 10.1109/JSAC.2004.839380.

[2] A. Goldsmith, S. Jafar, I. Maric, and S. Srinivasa, "Breaking Spectrum Gridlock With Cognitive Radios: An Information Theoretic Perspective," Proceedings of the IEEE, vol. 97, no. 5, 2009, pp. 894-914, doi: 10.1109/JPROC.2009.2015717.

[3] P. Fazio, M. Tropea, F. Veltri, and S. Marano, "A Novel Rate Adaptation Scheme for Dynamic Bandwidth Management in Wireless Networks," IEEE 75th Vehicular Technology Conference (VTC Spring), pp. 1-5, 2012, doi: 10.1109/VETECS.2012.6240289.

[4] S. Senthuran, A. Anpalagan, and O. Das, "Throughput Analysis of Opportunistic Access Strategies in Hybrid Underlay-Overlay Cognitive Radio Networks," IEEE Transactions on Wireless Communications, vol. 11, no. 6, pp. 2024-2035, 2012, doi: 10.1109/TWC.2012.032712.101209.

[5] I. F. Akyildiz, W.-Y. Lee, M. C. Vuran, and S. Mohanty, "NeXt generation/dynamic spectrum access/cognitive radio wireless networks: A survey," Computer Networks, vol. 50, no. 13, pp. 2127-2159, 2006, https: 10.1016/j.comnet.2006.05.001.

[6] Q. Zhang, J. Jia, and J. Zhang, "Cooperative relay to improve diversity in cognitive radio networks," IEEE Communications Magazine, vol. 47, no. 2, pp. 111-117, 2009, doi: 10.1109/MCOM.2009.4785388.

[7] A. A. Nasir, X. Zhou, S. Durrani, and R. A. Kennedy, "Relaying Protocols for Wireless Energy Harvesting and Information Processing," IEEE Transactions on Wireless Communications, vol. 12, no. 7, pp. 3622-3636, 2013, doi: 10.1109/TWC.2013.062413.122042.

[8] X. Zhou, R. Zhang, and C. K. Ho, "Wireless information and power transfer: Architecture design and rate-energy tradeoff," IEEE Transactions on Communications, vol. 61, no. 11, pp. 4754-4767, 2012, doi: 10.1109/TCOMM.2013.13.120855.

[9] M. Peng, S. Yan, and H. V. Poor, "Ergodic Capacity Analysis of Remote Radio Head Associations in Cloud Radio Access Networks," IEEE Wireless Communications Letters, vol. 3, no. 4, pp. 365-368, 2014, doi: 10.1109/LWC.2014.2317476.

[10] C. Jiang, N. C. Beaulieu, L. Zhang, Y. Ren, M. Peng, and H.-H. Chen, "Cognitive radio networks with asynchronous spectrum sensing and access," IEEE Network, vol. 29, no. 3, pp. 88-95, 2015, doi: 10.1109/MNET.2015.7113231.

[11] J. Mitola and G. Maguire, "Cognitive radio: making software radios more personal," IEEE Personal Communications, vol. 6, no. 4, pp. 13-18, 1999, doi: 10.1109/98.788210.

[12] J. Pastircak, J. Gazda, and D. Kocur, "A survey on the spectrum trading in dynamic spectrum access networks," Proceedings ELMAR-2014, 2014, doi: 10.1109/ELMAR.2014.6923334.

[13] T. Duong, V. Bao, and H.-J. Zepernick, "Exact outage probability of cognitive AF relaying with underlay spectrum sharing," Electronics Letters, vol. 47, no. 17, pp. 100-1002, 2011, doi: 10.1049/el.2011.1605. 
[14] M. Namdar and A. Basgumus, "Outage Performance Analysis of Underlay Cognitive Radio Networks with Decodeand-Forward Relaying," Cognitive Radio, 2017.

[15] Mondal, S., Dhar Roy, S., and Kundu, S., "Primary behaviour-based energy harvesting multihop cognitive radio network," IET Communications, vol. 110, no. 16, pp. 2466-2475, 2017, doi: 10.1049/iet-com.2017.0241.

[16] H. Song, J.-P. Hong, and W. Choi, "On the Optimal Switching Probability for a Hybrid Cognitive Radio System," IEEE Transactions on Wireless Communications, vol. 12, no. 4, pp. 1594-1605, 2013, doi:10.1109/TWC.2013.022213.120311.

[17] Tin, Phu Tran, Dang The Hung, Tan Nguyen, Tran Duy, and Miroslav Voznak, "Secrecy Performance Enhancement for Underlay Cognitive Radio Networks Employing Cooperative Multi-Hop Transmission with and without Presence of Hardware Impairments," Entropy, vol. 21, no. 2, pp. 217, 2019, doi:10.3390/e21020217.

[18] Tran, Hieu Dinh, Duy Trung Tran, and Seong Gon Choi, "Secrecy Performance of a Generalized Partial Relay Selection Protocol in Underlay Cognitive Networks," International Journal of Communication Systems, vol. 31, no. 17, 2018, doi: 10.1002/dac.3806.

[19] Bhowmick, Abhijit, Aindrila Chatterjee, and Tanya Verma, "Performance of DF Relaying in an Energy Harvesting Full Duplex Cognitive Radio Network," International Conference on Vision Towards Emerging Trends in Communication and Networking (ViTECoN), 2019, doi: 10.1109/vitecon.2019.8899470.

[20] Bhatnagar, M. R., "On the Capacity of Decode-and-Forward Relaying over Rician Fading Channels," IEEE Communications Letters, vol. 17, pp. 1100-1103, 2013, doi: 10.1109/LCOMM.2013.050313.122813.

[21] A. Ghasemi and E. Sousa, "Fundamental limits of spectrum-sharing in fading environments," IEEE Transactions on Wireless Communications, vol. 6, no. 2, pp. 649-658, 2007, doi: 10.1109/TWC.2007.05447.

[22] Tan N. Nguyen, T. H. Q. Minh, Phuong T. Tran and Miroslav Voznak, "Energy Harvesting over Rician Fading Channel: A Performance Analysis for Half-Duplex Bidirectional Sensor Networks under Hardware Impairments," Sensors, vol. 18, no. 6, pp. 1781, 2018, doi: 10.3390/s18061781.

[23] Gradshteyn, I. S., and I. M. Ryzhik, "Table of Integrals, Series, and Products," 2015.

[24] T. N. Nguyen, M. Tran, T.-L. Nguyen, D.-H. Ha, and M. Voznak, "Performance Analysis of a User Selection Protocol in Cooperative Networks with Power Splitting Protocol-Based Energy Harvesting Over Nakagami-m/Rayleigh Channels," Electronics, vol. 8, no. 4, p. 448, 2019, doi: 10.3390/electronics8040448.

[25] Tan N. Nguyen, T. H. Q. Minh, Phuong T. Tran, Miroslav Voznak, T. T. Duy, Thanh-Long Nguyen and Phu Tran Tin," "Performance Enhancement for Energy Harvesting Based Two-Way Relay Protocols in Wireless Ad-hoc Networks with Partial and Full Relay Selection Methods," Ad hoc networks, vol. 84, pp. 178-187, 2019, doi: 10.1016/j.adhoc.2018.10.005.

[26] Tan N. Nguyen, T. H. Q. Minh, Phuong T. Tran and Miroslav Voznak, "Adaptive Energy Harvesting Relaying Protocol for Two-Way Half Duplex System Network over Rician Fading Channel," Wireless Communications and Mobile Computing, 2018.

[27] Van-Duc Phan, Tan N. Nguyen, Minh Tran, Tran Thanh Trang, Miroslav Voznak, Duy-Hung Ha and Thanh-Long Nguyen, "Power Beacon-Assisted Energy Harvesting in a Half-Duplex Communication Network under Co-Channel Interference over a Rayleigh Fading Environment: Energy Efficiency and Outage Probability Analysis," Energies, vol. 12, no. 13, 2019, doi: 10.3390/en12132579.

[28] Tan N. Nguyen, Minh Tran, Duy-Hung Ha, Tran Thanh Trang and Miroslav Voznak, "Multi-Source in DF Cooperative Networks With PSR protocol Based Full-Duplex Energy Harvesting Over Rayleigh Fading Channel: Performance Analysis," Proceedings of the Estonian Academy of Sciences, vol. 68, no. 3, 2009, pp. 264-275, doi: 10.3176/proc.2019.3.03.

[29] Tan N. Nguyen, T. H. Q. Minh, Duy-Hung Ha, Thanh-Long Nguyen and Miroslav Voznak, "Energy harvesting based two-way full-duplex relaying network over Rician fading environment: performance analysis," Proceedings of the Estonian Academy of Sciences, vol. 68, no. 1, 2019, pp. 111-123, doi: 10.3176/proc.2019.1.11.

[30] A. Santamaria, P. Raimondo, M. Tropea, F. D. Rango, and C. Aiello, "An IoT Surveillance System Based on a Decentralised Architecture,” Sensors, vol. 19, no. 6, p. 1469, 2019, doi: 10.3390/s19061469. 